\title{
Expediente
}

DEPARTAMENTO DE MUSICA

Chefe: Prof. Dr. Leonardo Piermartiri

PROGRAMA DE PÓS-GRADUAÇÃO EM MÚSICA

Coordenador: Prof. Dr. Sérgio Figueiredo

\section{CENTRO DE ARTES}

Diretora: Profa. Dra. Gabriela Botelho Mager

UNIVERSIDADE DO ESTADO DE SANTA CATARINA

Reitor: Prof. Dr. Marcus Tomasi

\section{Editores}

Prof. Dr. Prof. Dr. Guilherme Sauerbronn de Barros

Profa. Dra . Teresa Mateiro

\section{Produção Editorial}

Maria Aparecida Clemencio

Maria Ermita Pereira

\section{Conselho Editorial}

Adriana Lopes Moreira (USP)

Ana Claudia Assis (UFMG)

Cristina Gerling (UFRGS)

Diósnio Machado (USP)

José Luiz Aróstegui (Universidade de Granada, Espanha)

Julie Ballantyne (University of Queesland, Austrália)

Liduino Pitombeira (UFRJ)

L. Poundie Burstein (CUNY - City University of New York)

Luiz Fiaminghi (UDESC)

Marcos Holler (UDESC)

Maria Bernardete Castelan Póvoas (UDESC)

Maria Cristina Carvalho de Azevedo (UnB)

Monica Vermes (UFES)

Patricia Adelaida González Moreno (Universidad Autónoma de Chihuaua)

Rafael Menezes Bastos (UFSC)

Silvio Ferraz (USP)

Viviane Beineke (UDESC)

Conselho de Pareceristas 
Acácio Piedade (UDESC)

Alberto T. Ikeda (UNESP)

Ana Guiomar Rêgo Souza (UFG)

Anternor Ferreira (Unb)

André Acastro Egg (UNESPAR)

Beatriz Magalhães Castro (UnB)

Denise Barata (UERJ)

Eduardo Lima Visconti (UNICAMP)

Eliane Tokeshi (USP)

Emerson Luiz De Biaggi (UNICAMP)

Guilherme Sampaio Garbosa (UFSM)

Helena Marinho (Universidade de Aveiro)

Hermilson Garcia do Nascimento (Unicamp)

Herom Vargas Silva (USCS)

Joana Cunha de Holanda (UFPel)

Luiz Otávio Braga (UNIRIO)

Luis Cláudio Barros (UDESC)

Luiz Guilherme Duro Goldberg (UFPel)

Luis Fernando Lazzarin ( UFSM)

Luzia Aurora Valeiro de Sousa Rocha (Universidade Lusíada e Universidade Nova de Lisboa/CESEM)

Luiz Otávio Rendeiro Corrêa Braga (UNIRIO)

Magda de Miranda Clímaco (UFG)

Marcos Câmara de Castro (USP)

Marcos da Cunha Lopes Virmond (USC)

Marcos Tadeu Holler (UDESC)

Marília Raquel Albornoz Stein (UFRGS)

Maurício Almeida Zamith (UDESC)

Norton Dudeque (UFPR)

Susana Bela Soares Sardo (Universidade de Aveiro)

Thais Nicolau (UDESC)

Vania Muller (UDESC)

Viviane Beineke (UDESC)

Zélia Chueke (UFPR)

\section{PRODUÇÃO GRÁFICA}

LabDesign

Projeto Gráfico

Ana Paula Lordello

Diagramação

Sarah Barros

Imagem da capa

Ana Paula Lordello 